comprising18.0\%(135/750) cervical lesions only, $11.7 \%$ (88/750) STI's only and $28.8 \%(216 / 750)$ both STI's and endocervical lesions. No abnormalities were detected in $19.9 \%$ of women $(149 / 750)$, while results from $21.6 \%(162 / 750)$ were missing. STI prevalence was 40.5\% (304/750); comprising HPV 20.0\%(150/750), candidiasis $16.1 \%(121 / 750)$, BV7.9\%(59/750), trichomonas vaginalis in $5.5 \%(41 / 750)$.Prevalence of endocervical abnormalities was 46.8\%(351/750); comprising Atypical Squamous Cells of Unknown Significance (ASCUS) 10.1\%(76/750); Cervical Carcinoma (CACX) $0.5 \%(4 / 750)$ and High(HGSIL) and Low (LGSIL) Grade Squamous Intra-epithelial Lesions of $12.1 \%(91 / 750)$ and $24.0 \%(180 / 750)$, respectively. The incidence HPV in this cohort was 24.8 per 100 women years (95\% CI: 15.7 to 37.2), incidence of ASCUS 7.8, HGSIL 11.1, LSIL 25.9, and CACX 0 per 100 women years, respectively. HPV was present in $19.2 \%$ of LSIL, $0.4 \%$ of HGSIL, $0.1 \%$ of patients with ASCUS and CACX.

Conclusion HPV infections and LGSIL were the dominant genital tract abnormalities in TB-HIV co-infected patients accessing ART.

\section{P3.234 PREVALENCE AND ESTIMATED HIV-1 INCIDENCE AT TWO VOLUNTARY COUNSELING AND TESTING CENTERS IN NORTHEAST OF BRAZIL}

doi:10.1136/sextrans-2013-051184.0691

'K 0 Lima, ${ }^{2}$ A Salustiano Cavalcanti, ${ }^{3} \mathrm{D}$ M Salustiano, ${ }^{2} S$ P Silva, ${ }^{4} \mathrm{R} S$ Diaz, ${ }^{1} \mathrm{H}$ R Lacerda. 'Universidade Federal de Pernambuco -UFPE, Recife -Pernambuco, Brazil; 'Laboratório Central de Saúde Pública de Pernambuco, Recife -Pernambuco, Brazil; ${ }^{3}$ Voluntary Counseling and Testing Centers Herbert de Souza, Cabo de Sto. Agostinho -Pernambuco, Brazil; ${ }^{4}$ Universidade Federal de São Paulo - UNIFESP, São Paulo, Brazil

Background Estimates of incidence and prevalence are important tools for the investigation of regional trends of the HIV epidemic. Currently, the use of immunoassays for epidemiology investigation is referred by RITA (Recent Infection Testing Algorithm). This study aimed to determine the prevalence HIV-1 incidence in two Voluntary and Counseling Testing (VCT) Centers, in the Metropolitan Region of Recife - Northeast of Brazil, in the period from 2008 to 2009

Methods Demographics and behavioural data were obtained from 245 individuals diagnosed as HIV-positive among 19,451 volunteers screened from January 2008 to December 2009. The BED - CEIA was used for the determination of recent infection and estimate HIV-1 incidence.

Results HIV-1 prevalence was $1.3 \%$ (95\% CI: $1: 14$ to $1: 46)$ and the corresponding HIV-1 estimated incidence was $0.71 \%$ /year $(95 \% \mathrm{CI}$ : $0.53-0.89)$. The males obtained a higher prevalence $(2.6 \%, 95 \% \mathrm{CI}$ : $2: 13$ to $3: 07)$ and incidence $(1.29 \% / y e a r, 95 \%$ CI: $0.79-1.79)$ than females, whose prevalence was $0.8 \%$ (95\% CI: $0.62-0.98)$ and the incidence rate was $0.52 \%$ year (95\% CI: $0.34-0.70)$. A high rate of recent infection was observed in both genders (male: $25 \%$ female: $29.9 \%$ ).

Conclusions Our study shows a high rate of recent infection for HIV-1 in genders, as well as a high prevalence and incidence among males, indicating that prevention strategies in this population should be intensified.

\section{P3.235 GLOBAL ECOLOGICAL STUDY OF HIV AND HSV-2 PREVALENCE}

doi:10.1136/sextrans-2013-051184.0692

'S P Kouyoumjian, ' 'G R Mumtaz, ${ }^{2}$ P Vickerman, ${ }^{1,3,4} \mathrm{~L}$ J Abu-Raddad. 'Weill Cornell Medical College-Oatar, Qatar Foundation, Education City, Doha, Oatar; ${ }^{2}$ London School of Hygiene and Tropical Medicine, London, UK; ' Weill Cornell Medical College, Cornell University, New York, NY, United States; ${ }^{4}$ Fred Hutchinson Cancer Research Center, Seattle, WA, United States
Background HIV and herpes simplex virus type-2 (HSV-2) are infections transmitted predominantly through sexual intercourse. We explored the ecological association between the prevalence of HIV and HSV-2 among human populations through a global review. Methods We conducted a global systematic literature review of HIV and HSV-2 prevalence following the PRISMA guidelines. Sources of data used were Medline (PubMed) and Embase databases, and several country-level reports. No language, country, or year limitations were imposed. We included any publication with a serological measurement of HIV and HSV-2 prevalence in the same study population.

Results A total of 2,927 records were screened. Based on preliminary descriptive analysis, we found that HIV prevalence increased steadily with HSV-2 prevalence in all populations where the dominant mode of transmission was sexual. HSV-2 prevalence was consistently larger than HIV prevalence. Overall, among high-risk populations, both infections prevalence was high. Among general population groups, HIV prevalence varied across settings, but was correlated with HSV-2 prevalence, which also varied widely. Though HIV and HSV-2 prevalence levels were correlated across populations, there were settings with very low HIV prevalence regardless of HSV-2 prevalence. For children and injecting drug users where the dominant mode of HIV transmission was not sexual, there was no apparent ecological association between the two infections.

Conclusions Our findings support a strong ecological association between HIV and HSV-2 prevalence in all populations where the dominant mode of HIV transmission is sexual. Sexual networks conducive of HSV-2 transmission appear to be also conducive of HIV transmission. Further analytical work is needed to quantify the ecological association between the two infections, to determine whether HSV-2 could be predictive of HIV epidemic potential, and to assess whether there is a threshold of HSV-2 prevalence necessary for a sexual network to be sustainable for HIV transmission besides HSV-2 transmission.

\section{P3.236 ANALYSIS OF RISK FACTORS INCIDENCE OF SEXUALLY TRANSMITTED INFECTIONS IN THE WOMEN INDIRECT SEX WORKERS IN MATARAM CITY, INDONESIA, 2012}

doi:10.1136/sextrans-2013-051184.0693

K M Kadek. Health Office, Mataram, Indonesia

Background Sexually transmitted infections are a public health problem in all countries, including Indonesia. The estimated number of people exposed to a sexually transmitted infection that can be treated approximately more than 30 million cases annually. In Mataram City 2011, found as many as 896 new cases of sexually transmitted infections. Women indirect sex workers have an important role in the spread of sexually transmitted infections and HIVAIDS cases increased.

Research Methods Design study was a cross sectional with a total sample of 66 women indirect sex workers. Risk factor of STIs is age of first sex, ever having sex, the number of customers per day, use of condoms, clean hands after sex, change underwear after sex, alcohol consumption habits and the habit of consuming drugs. Potential risk factors were explored using a structured questionnaire of the month from May to June 2012. Data were analysed using, bivariable and multivariable statistics.

Results From the bivariable analysis, risk factors for STIs were ever having sex (OR 2.33, CI 5.15-1:05), not using condoms (OR 3.13, CI: 1.36 to 7.20 ) and the number of customers per day (OR 2.60, CI: 1.13 to 6.01). Multivariable analysis showed that the risk factors that influence the incidence of sexually transmitted infections are not using condoms (adjusted OR 6.55, CI 1.83 to 23, 43) and the number of customers per day (adjusted OR 5.01, CI 1.41 to 18.29) 
Conclusion Not using a condom in serving customers in women indirect sex workers be risk factors for STIs. Need an effort to monitor the prevalence of sexually transmitted infections continued cooperation and support of stakeholder in pressing the prevalence of STIs

\section{P3.237 AN ASSESSMENT OF STD SCREENING PRACTICES IN NEW PATIENTS ATTENDING AN HIV PRIMARY CARE CLINIC}

doi:10.1136/sextrans-2013-051184.0694

R Blanchard, J Keller, A Wilkin, L Bachmann. Wake Forest University Health Sciences, Winston-Salem, NC, United States

Background Since 2003, national guidelines recommend that all new HIV patients undergo screening for sexually transmitted diseases (STD) during their initial visit. We evaluated STD screening practises in new patients attending an HIV Primary Care Clinic in North Carolina.

Methods This retrospective cohort study included HIV-infected patients greater than 18 years of age enrolled in the clinic between January 1, 2008 and December 31, 2010. Data on rapid plasma reagin (RPR) testing for syphilis and Neisseria gonorrheoeae (GC) and Chlamydia trachomatis (CT) testing by site of exposure, within 1 year of the new patient visit, were extracted via chart review to examine comprehensiveness of STD screening practises.

Results Between 2008 and 2010, 693 (68\% male and 32\% female) new patients entered care, most of whom were screened for syphilis within one year of the new patient visit $(94.5 \%$ in $2008,96.6 \%$ in $2009,96.9 \%$ in $2010, p=0.41)$. In contrast, only $21.3 \%$ were tested for GC and CT within one year of the first visit in 2008, $34.3 \%$ in 2009 , and $54.3 \%$ in 2010 ( $p=<0.0001$ ). Of those screened, the prevalence of past/current syphilis ranged from $8.4-10.1 \%(p=0.81)$ while positive testing for either GC or CT ranged from $2.3-9.1 \%$ $(p=0.20)$. In 2008, 1 rectal and 1 pharyngeal GC and CT test was performed versus 9 rectal and 8 pharyngeal tests in 2009 and 20 rectal and 14 pharyngeal tests in 2010. Of these, 13.3\% of rectal tests and $8.7 \%$ of pharyngeal tests were positive, in contrast to $3.7 \%$ of genital tests.

Conclusion While screening practises improved from 2008 to 2010, new HIV patients continue to be under-screened for gonorrhoea and chlamydia, particularly at extra-genital sites. This deficiency is particularly important as, of those screened, prevalence was higher in extra-genital compared to genital sites. Efforts should be made to increase routine extra-genital screening.

\section{P3.238 GENERATING EVIDENCE THROUGH SEROSURVEILLANCE; HELPING IN PROGRAMMIE DESIGNING TO MITIGATE SEXUALLY TRANSMITTED INFECTIONS (STIS) AMONG FEMALE SEX WORKERS (FSWS) IN BANGLADESH}

doi:10.1136/sextrans-2013-051184.0695

'M Amin, ${ }^{2}$ M A Rahman, 'A F Khuda, 'S Rasin, 'L Rahman, 'F Sultana, 'M S Islam. 'Save the Children, Dhaka, Bangladesh; 'National AIDS/STD Programme, Dhaka, Bangladesh

Background The overall prevalence of HIV and active syphilis are $0.7 \%$ and $3 \%$ respectively revealed in 9th serosurveillance among most at risk population. High active syphilis rates suggest practise of unsafe sex and a surrogate marker of unsafe sex is active syphilis. STIs and HIV are linked as they share the same risk behaviour, STIs facilitate acquisition and transmission of HIV, and some STI pathogens become more virulent in presence of HIV-related immunodeficiency. Under Global Fund support and initiative Save the Children stepped in (2008) with essential service (primly STI management, Condom distribution, BCC session through DICs and outreach) for FSWs nationwide in 51 districts which includes all serosurveillance sampling sites for FSWs.
Methods 8th round serosurveillance was conducted between July and December 2007 where total 4797 FSWs in 15 cities were sampled. 9th serosurveillance was conducted between December 2010 to June 2011 where total 3568 FSWs were sampled from 13 cities of Bangladesh. Same sampling methodology followed in both the rounds. Sero-surveillance sampling site were under implementation coverage.

Results In 8th sero-surveillance, $>5 \%$ active syphilis was detected in five sites namely street FSWs of Chittagong, Rangpur, Dhaka, hotels FSWs of Sylhet (8.3\%) and casual FSWs in Chandpur. In 9th serosurveillance, $>5 \%$ active syphilis was detected in three sites, street FSWs of Hili, Chittagong and hotel FSWs of Sylhet. Active Syphilis rate decreased in all the 5 sites except hotel FSWs in Sylhet (9.3\%). Moreover, there is decreasing trends of active syphilis among FSWs in several sites in 9th round comparing to 8th.

Conclusion High active syphilis rate highlight the need for ongoing programme intensification to decline STI trend. Evidence with good programmatic implication extrapolated and translated through serosurveillance could tailor-made the ongoing intervention and also designing the future programme.

\section{P3.239 HIV AND SYPHILIS INFECTION AND RISK BEHAVIOURS AMONG FEMALE SEX WORKERS IN PARAGUAY IN 2012}

doi:10.1136/sextrans-2013-051184.0696

'G Aguilar, ${ }^{2} \mathrm{~J}$ Jacobson, 'L Gimenez, ${ }^{1} \mathrm{~A}$ Barboza, ${ }^{1} \mathrm{~A}$ Kawabata. ${ }^{1}$ National Program of Control of HIVAidsSTI, Asuncion, Paraguay, ${ }^{2}$ Independent Consultant, Bogota, Colombia

Introduction Syphilis is an important public health problem in Paraguay. A 2006 study found high levels of syphilis (19.0\%) infection and unprotected sex with most recent client (also 19.0\%) and moderate level of HIV infection (1.8\%) in female sex workers (FSW). Several HIV and STI prevention interventions have been put in place since then. A recent study sought to assess current infection and risk behaviours.

Methods 432 FSW were recruited from sex work locations in the greater metropolitan area of Asuncion using time-location sampling between January and May, 2012. A face-to-face survey and rapid and confirmatory biological tests in accordance with national algorithms assessed risk behaviours and syphilis and HIV infections. Weighted statistical analysis accounted for the sampling strategy and adjusted for clustering by recruitment venue. Adjusted $95 \%$ confidence intervals (CI's) for the 2006 and 2012 studies were compared to identify significant trends.

Results Median age of participants was 25 years (interquartile range (IOR), 21-32). Median age of first sex work was 20 years (IOR 18-24). Differences in VIH prevalence among 2006 (1.8\%) and 2012 $(2.5 \% \%)$ studies weren't significant $(P=0.4)$. Prevalence syphilis $(25.3 \%$, IC $21.3 \%-29.8 \%)$ were higher than 2006 estimates $(P<0.05))$. Median number of clients during the past week was 15 (IOR 8-25). 40.5\% (CI 35.8-45.3) reported drinking at last sex with a client. Condom use with last client was $98.1 \%$ (IC 96.2\%-99.1\%), increased over $2006(P<0.05)$ Among 59.3\% who had a stable partner, $77.3 \%$ (IC 71.4-82.0) had not used a condom at last sex with a stable partner.

Conclusion Increasing levels of syphilis infection suggest a need to reevaluate and intensify STI prevention and control interventions, with greater emphasis on diagnosis and treatment, reducing drinking with clients as well as providing alternatives to sex work for FSW.

\section{P3.240 DOES THE PREVALENCE OF SEXUALLY TRANSMITTED DISEASES ADEQUATELY REFLECT SEXUAL TRANSMISSION OF HEPATITIS C IN THE HIV-INFECTED POPULATION?}

doi:10.1136/sextrans-2013-051184.0697 